\title{
BER Evaluation of a Passive SOI WDM Router
}

\author{
Alberto Parini, Gaetano Bellanca, Andrea Annoni, Francesco Morichetti, Andrea Melloni, Michael J. Strain, \\ Marc Sorel, Mathilde Gay, Christelle Pareige, Laurent Bramerie, and Monique Thual
}

\begin{abstract}
The performance of a ring-resonator based passive wavelength router, suitable for optical networking at chip level, is evaluated through BER measurements in single channel $10 \mathrm{Gbit} / \mathrm{s}$ and 3-channel $10 \mathrm{Gbit} / \mathrm{s}$ WDM configurations. As the routing path involves a different number of routing elements, depending on the wavelength used for the propagating signal, the performance of three output ports with respect to a specified input one are experimentally evaluated and discussed. Measurements show that low rejection on the filter elements due to fabrication issues mostly affect the channel on the through path with respect to the dropped ones.
\end{abstract}

Index Terms-Optical switching devices, photonic integrated circuits, optical interconnections, microring resonators, Silicon on Insulator, Bit-Error-Rate.

\section{INTRODUCTION}

$\mathrm{C}$ HIP multiprocessor architectures represent the state of the art in the design of high performance digital integrated systems. Currently, silicon technology allows the manufacturing of hundreds of computational cores on the same circuit die, hence reaching a high grade of parallelism and scalability. Due to the large number of interconnected functional entities, the data exchange among them can only be implemented by means of a shared network infrastructure (Network on Chip - NoC). As the number of linked cores increases, the burden of the communication infrastructure in

Manuscript received August 9, 2013; revised September 14, 2013; accepted October 1, 2013. This work is supported by the Italian Ministry of the University and Research through PHOTONICA FIRB 2008 and SAPPHIRE PRIN 2009 Projects. Alberto Parini thanks the Programma Operativo FESR 2007-2013 of the Emilia Romagna Region - Attività I.1.1 for the financial support to his research activity. We acknowledge the support from the technical staff of the JWNC at Glasgow University.

A. Parini is with the MIST E-R Laboratory, Via Saragat 1, 44122 Ferrara, Italy, and with the Department of Engineering, University of Ferrara, Via Saragat 1, 44122 Ferrara, Italy (e-mail: alberto.parini@unife.it).

G. Bellanca is with the Department of Engineering, University of Ferrara, Via Saragat 1, 44122 Ferrara, Italy (e-mail: gaetano.bellanca@unife.it).

A. Annoni, F. Morichetti and A. Melloni are with the Dipartimento di Elettronica, Informazione e Bioingegneria, Politecnico di Milano, Via Ponzio 34/5, 20133 Milano, Italy (e-mail: andrea.annoni@gmail.com; morichetti@elet.polimi.it; melloni@elet.polimi.it).

Michael J. Strain was previously with the School of Engineering, University of Glasgow, Glasgow G128LT, U.K., and currently with the Institute of Photonics, University of Strathclyde, UK. (email: Michael.strain@strath.ac.uk).

M. Sorel is with the School of Engineering, University of Glasgow, Glasgow G128LT, U.K. (e-mail: Marc.Sorel@glasgow.ac.uk).

M. Gay, C. Pareige, L. Bramerie and M. Thual are with the CNRS-Foton Laboratory (UMR 6082), Enssat, Université Européenne de Bretagne (UEB), BP 80518, 22305 Lannion, France (e-mail: mathilde.gay@enssat.fr; Christelle.Pareige@enssat.fr; bramerie@enssat.fr; thual@univ-rennes1.fr).

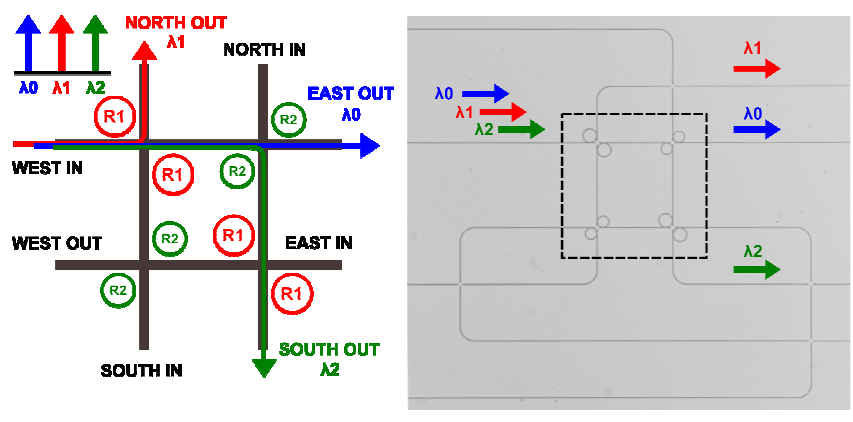

Fig. 1. Left: sketch of the characterized GWOR topology with four input and four output ports. The wavelength-dependent routing is achieved through the choice of different radii for rings R1 and R2. Right: top-down microscope image of the fabricated device. The highlighted region is the switching core of the router. In the real device, to ease measurements, the input ports are all positioned on the left side of the chip whereas the output ones are on the right. For this reason, some optical circuitry (bend, crossing) have been added in the final layout.

terms of bandwidth demand and power consumption becomes predominant, such as to constitute the actual upper bound to a further expansion of the system. To overcome this limit, an attractive solution is to realize optical-based interconnections among the cores (Optical Network-on-Chip (ONoC)) [1]. Indeed, photonic-based systems can achieve extremely high bandwidths through wavelength division multiplexing (WDM), while maintaining a sustainable power dissipation level [2].

In ONoCs literature, router topologies are usually classified into two families: active and passive networks. In active networks all rings have the same radius, and their resonances are tuned via thermo-optic or electro-optic effects. This allows a dynamic "on the fly" allocation of the routing paths, but requires extra tuning circuitry. In passive networks, on the contrary, the routing paths are set once during design, by suitably placing sets of rings with different radii. Passive solutions reduce the technological complexity of the circuit, by avoiding the presence of tuning mechanisms, but impose stricter constraints at architectural and system levels.

While active topologies have been extensively analyzed $[3,4,5]$, in this contribution we report on the Bit Error Rate (BER) characterization of a passive topology with four input and four output ports, realized in Silicon-on-Insulator (SOI) technology. The switching capabilities are rated through BER curves for each significant routing path in a $10 \mathrm{Gbit} / \mathrm{s}$ single channel configuration and in a 3 -channel $(3 \times 10 \mathrm{Gbit} / \mathrm{s}) \mathrm{WDM}$ operating mode. The single channel BER, measured by exciting only one channel, allows to evaluate loss in performance due to the propagation in the component. On the other hand, WDM BER assesses the robustness of the device with respect to crosstalk effects when several channels 
propagate together, thus giving a direct estimation of the discrimination capability of the router [6].

The article is structured as follows: section II describes the main operating characteristics of the investigated device, with fabrication details highlighted in section III. In section IV, the setup used for BER measurements is illustrated. Finally, results are reported and discussed in section V.

\section{DESCRIPTION AND OPERATION}

Fig. 1 shows the topology of the characterized four input, four output Generic Wavelength-routed Optical Router $(4 \times 4$ GWOR) structure [7]. The device consists of four pairs of rings, positioned at the corners of a square network layout (see Fig. 1). The rings labeled R1 have a slightly different round trip length (i.e. different resonant wavelength) with respect to the ones labeled $\mathrm{R} 2$. This spectral interleaving in the $1550 \mathrm{~nm}$ wavelength range provides the routing assignment of Tab 1.

Tab. 1: Routing table of the $4 \times 4$-GWOR

\begin{tabular}{|c|c|c|c|c|}
\hline & $\begin{array}{c}\text { North } \\
\text { Out }\end{array}$ & $\begin{array}{c}\text { East } \\
\text { Out }\end{array}$ & $\begin{array}{c}\text { South } \\
\text { Out }\end{array}$ & $\begin{array}{c}\text { West } \\
\text { Out }\end{array}$ \\
\hline North In & - & $\lambda_{2}$ & $\lambda_{0}$ & $\lambda_{1}$ \\
\hline East In & $\lambda_{2}$ & - & $\lambda_{1}$ & $\lambda_{0}$ \\
\hline South In & $\lambda_{0}$ & $\lambda_{1}$ & - & $\lambda_{2}$ \\
\hline West In & $\lambda_{1}$ & $\lambda_{0}$ & $\lambda_{2}$ & - \\
\hline
\end{tabular}

As the device is topologically invariant under step rotations of $180^{\circ}$ (see Fig. 1), and orthogonal input ports differ only with regards to the first encountered ring resonator routing wavelength, we have decided to characterize the device using the West-In port.

By injecting in this input port three signals with different wavelengths, namely $\lambda_{0}, \lambda_{1}$ and $\lambda_{2}$, the routing paths inside the device are the following:

1. $\lambda_{0}$ is a through (out of resonance) wavelength for both R1 and $\mathrm{R} 2$ ring pairs; consequently, the signal at this wavelength is directly routed from West-In to East-Out (through path);

2. $\lambda_{1}$ is a drop wavelength for the $\mathrm{R} 1$ rings; the associated data stream is then redirected toward the North-Out port (drop path driven by $\mathrm{R} 1$ rings);

3. $\lambda_{2}$ is a drop wavelength for the R2 ring family, but not for the R1 one; the associated data stream is therefore routed to the South-Out port (drop path driven by $\mathrm{R} 2$ rings).

In this device, U-turn paths, i.e. West-In to West-Out, are topologically forbidden (a computational core, in fact, does not need to communicate with itself).

\section{DEVICE FABRICATION AND CHARACTERIZATION}

The proposed integrated GWOR network was fabricated on a commercial Silicon-on-Insulator (SOI) wafer with a $220 \mathrm{~nm}$ thick silicon layer on a $2 \mu \mathrm{m}$ thick oxide buffer layer. The channel waveguide was patterned using electron-beam lithography on a hydrogen silsesquioxane (HSQ) resist, followed by inductively coupled plasma etching [8]. The silicon core has a width of $480 \mathrm{~nm}$ and is buried under an oxide bi-layer made of $550 \mathrm{~nm}$-thick HSQ layer and $350 \mathrm{~nm}$-thick $\mathrm{SiO}_{2}$ films, respectively. Propagation loss is about $3 \mathrm{~dB} / \mathrm{cm}$ for $\mathrm{TE}$ polarization at a wavelength of $1550 \mathrm{~nm}$. Inverse tapered waveguide sections buried in a SU-8 polymer waveguide were realized at the chip's end facets. These mode adapters reduce the coupling loss with tapered lensed fibers to $(5 \pm 1 \mathrm{~dB}) /$ facet as well as the spurious Fabry-Perot resonance effects due to optical back-reflections at waveguide terminations.

Circuit design and mask generation was produced by using a commercial simulator [9] exploiting a building block approach

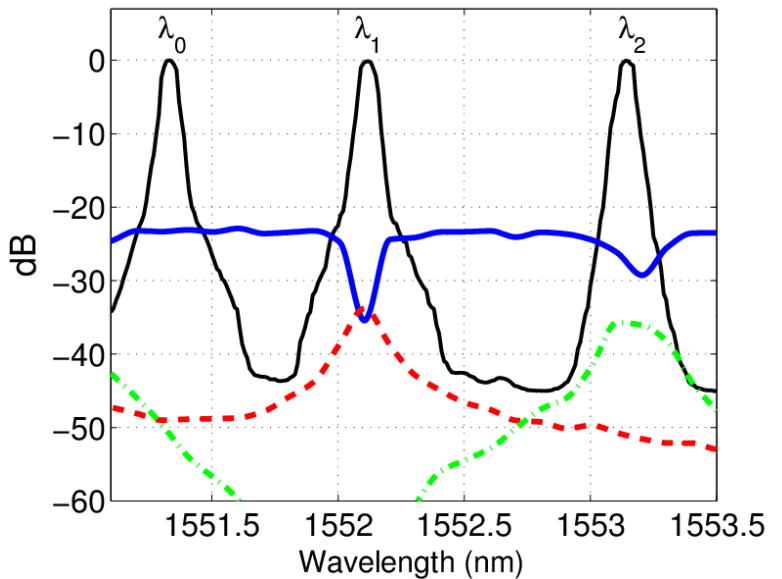

Fig. 2. Spectra of the three output ports of the device (blue solid line for East-Out, red dashed line for North-Out and green dash-dotted line for South-Out), together with the spectra of the three laser sources used for the BER estimations (black thin solid line).

for photonic integrated circuits [10]. The bending radius of the resonators of the GWOR is $20 \mu \mathrm{m}$ for rings $\mathrm{R} 1$ and $18 \mu \mathrm{m}$ for rings $\mathrm{R} 2$, resulting in a free spectral range FSR of $552 \mathrm{GHz}$ and $613 \mathrm{GHz}$, respectively. The finesse of all the rings is about 35 , corresponding to a drop port transmission bandwidth of 15.5 GHz (R1) and $17.5 \mathrm{GHz}$ (R2). The bus-to-ring power coupling $\mathrm{K}=7.5 \%$ is obtained through zero-length directional couplers with a gap distance of $150 \mathrm{~nm}$ between the straight bus waveguide and the bent waveguide of the ring. The waveguide crossings of the GWOR are optimized by introducing linear-shaped tapers with an overall footprint of $15 \times 15 \mu \mathrm{m}^{2}$, in order to reduce both insertion losses $(<0.7 \mathrm{~dB})$ and cross-talk between orthogonal waveguides $(<-30 \mathrm{~dB})$. The overall footprint of the GWOR device is about $0.8 \mathrm{~mm}^{2}$.

\section{BER EVALUATION SETUP}

Fig. 2 shows the measured transmission spectra of the three output ports in the spectral range $1551 \mathrm{~nm}-1553.5 \mathrm{~nm}$. Within this range, three possible carrier wavelengths corresponding to the previously described routing paths of the device are $\lambda_{0}=1551.32 \mathrm{~nm}$ (through path between the West-In and the East-Out ports), $\lambda_{1}=1552.11 \mathrm{~nm}$ (resonance of the R1 pairs) and $\lambda_{2}=1553.13 \mathrm{~nm}$ (resonance of the R2 pairs). On the same figure, the black solid thin line shows the spectra of the three laser sources tuned at $\lambda_{0}, \lambda_{1}$ and $\lambda_{2}$, respectively. As can be observed from Fig. 2, the total losses (path losses + facet losses) measured at each channel wavelength are $23.3 \mathrm{~dB}$ at $\lambda_{0}$, 
$33.78 \mathrm{~dB}$ at $\lambda_{1}$ and $35.81 \mathrm{~dB}$ at $\lambda_{2}$. As previously stated, the BER of each channel is measured in two different configurations: single channel and 3-channel WDM.

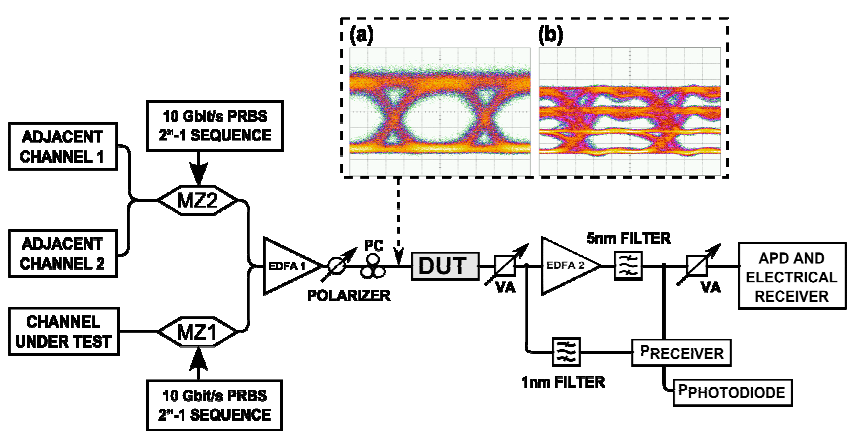

Fig. 3. Experimental setup for BER evaluation in single channel and WDM configuration. Inset (a) shows the eye diagram of the input signal when operating in single channel configuration, whereas inset (b) shows the eye diagram of the input signal in WDM configuration.

In the single channel scenario, only the carrier wavelength which corresponds to the path under test is launched into the West-In port of the DUT. In the WDM configuration, on the contrary, the BER for each output port is evaluated by injected simultaneously into the West-In port of the DUT a comb of three contiguous wavelengths associated with the three routing paths of the device.

Fig. 3 shows in details the experimental setup employed for the BER measurements in the WDM configuration [11]. It must be pointed out that the setup for the single channel measurement is the same, except that in this case only the source pertinent to the examined path (channel under test) is active.

When operating in WDM configuration, two of the three wavelengths coming from the laser sources are combined with a $3 \mathrm{~dB}$ coupler and sent to the Mach-Zehnder modulator MZ2; the other channel, on the contrary, is sent to MZ1. The modulators receive two $10 \mathrm{Gbit} / \mathrm{s}$ binary NRZ input streams of $2^{31}-1$ Pseudo Random Bit Sequences (PRBS). MZ1 modulates the channel under test (in turns, $\lambda_{0}, \lambda_{1}$ and $\lambda_{2}$ ), whereas MZ2 modulates the adjacent two interfering channels (in turns, the pairs $\left(\lambda_{1}, \lambda_{2}\right),\left(\lambda_{0}, \lambda_{2}\right)$, and $\left.\left(\lambda_{0}, \lambda_{1}\right)\right)$.

This two modulator-based configuration guarantees that the data stream of the channel under test is not correlated to the data stream of the other two interfering channels. A further $3 \mathrm{~dB}$ coupler merges these signals before passing them through an erbium doped fiber amplifier (EDFA1). After the amplification stage, the signals are adjusted on a TE polarized mode by a Polarization Controller (PC) to avoid excitation of TM resonances, and finally coupled into the DUT. Microlensed fibers, with a mode field diameter of $2 \mu \mathrm{m}$, provide an efficient input and output coupling to the DUT.

Moreover at the injection side, when operating in WDM, the power magnitude of the three wavelengths is kept equalized, in order to make the router to operate with a balanced comb.

\section{RESULTS AND DISCUSSION}

We evaluated the power penalty in single channel and in
WDM configurations for a Bit Error Rate ranging between $10^{-4}$ and $10^{-10}$, with respect to the Back to Back (B2B) experiment.
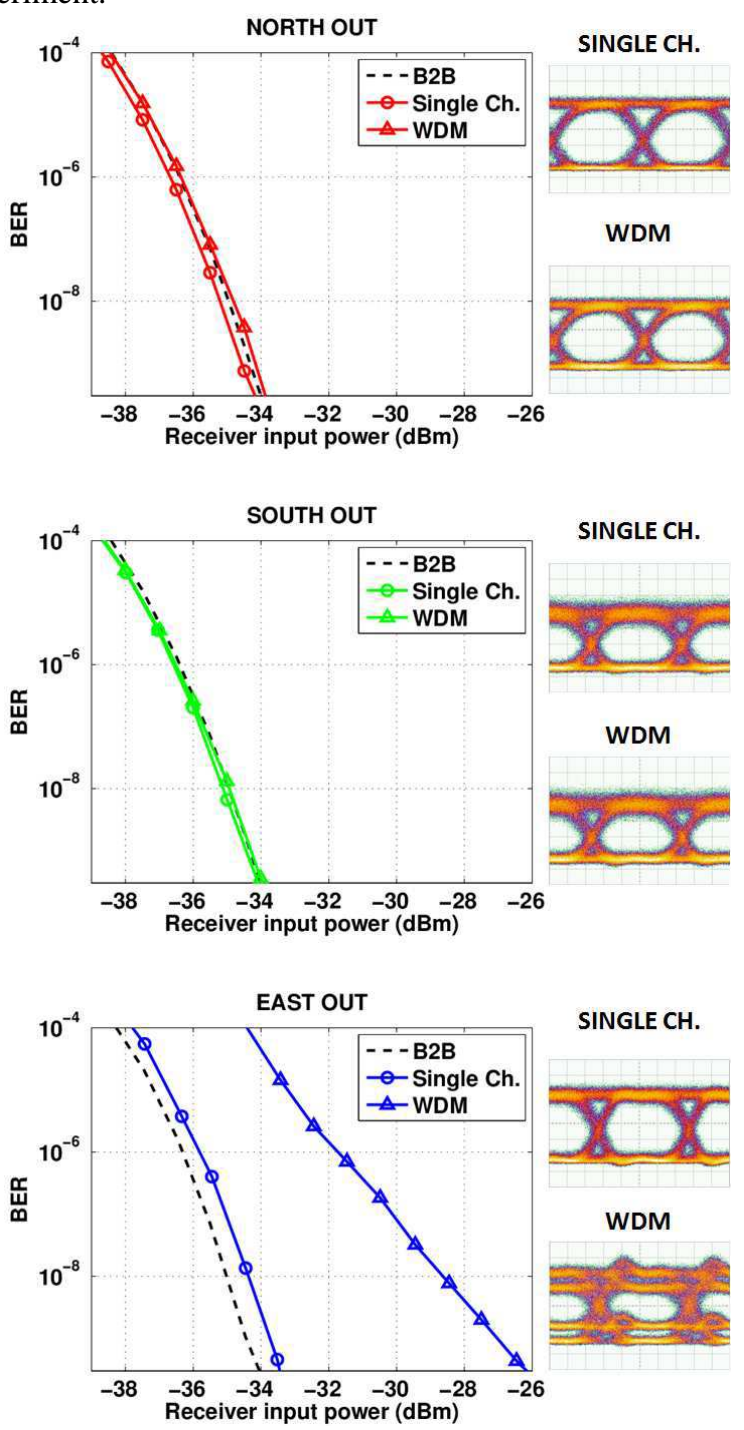

Fig. 4. BER curves in single channel and in 3-channel WDM configurations for the three output ports of the device. Beside each plot the eye-diagrams corresponding to both scenarios are reported.

In the B2B experiment, the DUT is replaced with an optical loss stage, whose total attenuation matches the one introduced by the DUT on the observed port. Consequently, the B2B BER is a measure of the power penalty caused by the degradation of the optical signal to noise ratio, due exclusively to intrinsic losses. Results obtained for the different routing paths are summarized in the following:

NORTH OUT: This is a drop path driven by the R1 ring resonances. The routing wavelength associated with this port is $\lambda_{1}$, while $\lambda_{0}$ and $\lambda_{2}$ represent the interfering signals when operating in WDM configuration. The top left panel in Fig. 4 shows the BER curves obtained in single channel (red line with circles) and in WDM (red line with triangles) operation, respectively. As one can note, the single channel configuration performs better than the corresponding B2B experiment (black dashed line). This apparently counterintuitive effect should not 
be surprising; in fact, since the device under test is a cascade of resonant filters, it can slightly modify the shape of the propagating pulses due to the filter selectivity, and give a better adaptation of the signal to the receiver, with a consequent increase of the sensitivity. Actually, this effect takes place for the north-out port and the south-out port, whose routing paths are associated with a drop transit (filtering) in rings $\mathrm{R} 1$ and $\mathrm{R} 2$, respectively. At a BER of $10^{-9}$, the power penalty measured in the WDM configuration with respect to the single channel one is only $0.4 \mathrm{~dB}$; therefore, it is possible to assert that the associated routing path is only affected by intrinsic penalties due to the propagation, and is substantially robust with respect to crosstalk from the other channels. Moreover, the eye diagrams in both operating modes are nearly coincident, except for a slightly higher noise level affecting the eye in the WDM case.

SOUTH OUT: This drop path is driven by the $\mathrm{R} 2$ ring resonances. The associated wavelength is now $\lambda_{2}$ (right peak in the spectrum of the signal presented in Fig. 2), whereas $\lambda_{0}$ and $\lambda_{1}$ are the interfering WDM signals. From the measured transmission spectrum of Fig. 2 (green dash-dotted line) one can see that the rejection of $\lambda_{0}$ with respect to $\lambda_{2}$ is $15 \mathrm{~dB}$, whereas the rejection of $\lambda_{1}$ is more than $25 \mathrm{~dB}$. As a consequence of the high selectivity of this channel, the crosstalk can be considered negligible. This is confirmed by the corresponding WDM BER curve, which does not show any significant penalty with respect to the one measured in single channel configuration (only $0.14 \mathrm{~dB}$ at a BER of $10^{-9}$ ). The robustness with respect to crosstalk is pointed out also by the eye diagrams, which are fully open in both cases.

EAST OUT: Unlike the previous two, this output port is a through-path for both $\mathrm{R} 1$ and $\mathrm{R} 2$ ring families. The associated wavelength is now $\lambda_{0}$, while $\lambda_{1}$ and $\lambda_{2}$ are the interfering WDM signals. The BER of the single channel shows no significant penalties with respect to the B2B experiment, as claimed by the corresponding eye diagram. On the contrary, when operating in WDM regime, the BER curve exhibits a power penalty of $7 \mathrm{~dB}$, (for a BER of $10^{-9}$ ), which is induced by the strong crosstalk of the interfering channels. It must be noted that the low performance on this path is determined by the poor rejection of the north-eastern R2 ring couple. In fact, by observing the spectrum in Fig. 2 (blue solid line) one can note that the relative rejection of $\lambda_{2}$ with respect to $\lambda_{0}$ is of only $3 \mathrm{~dB}$ (right notch in the same figure). Due to this poor rejection, a high fraction of the $\lambda_{2}$ signal is not filtered out and remains on the bus waveguide, giving rise to interference on this output. This effect is also confirmed by the presence of multiple tracks in the eye diagram of Fig. 4. To assure a better performance on this path, R2 nodes must be precisely tuned. To this purpose, photo-induced treatments $[12,13]$ can be exploited for a post-fabrication compensation of fabrication tolerances, as well as to trim the circuit response.

\section{CONCLUSION}

The switching capabilities and the robustness to crosstalk of a four input - four output GWOR device realized in SOI technology have been evaluated through BER measurements in single $(10 \mathrm{Gbit} / \mathrm{s})$ and 3 channel $(3 \times 10 \mathrm{Gbit} / \mathrm{s})$ WDM configurations. Comparisons between BER performance in single and multichannel operative conditions enable a clear understanding of the main sources of transmission quality deterioration in the system, pointing out the most critical paths across the router. Indeed, results show that the poor rejection of a switching node due to fabrication issues introduces a crosstalk power penalty of $7 \mathrm{~dB}$ on the through path. Surprisingly, the drop path driven by the faulty node is not penalized (power penalties lower than $0.5 \mathrm{~dB}$ ), thus highlighting the stronger robustness of drop-port routed channels to crosstalk effects.

\section{REFERENCES}

[1] A. W. Poon, X. Luo, F. Xu, and H. Chen, "Cascaded MicroresonatorBased Matrix Switch for Silicon On-Chip Optical Interconnection", Proceedings of the IEEE, vol. 97, no.7, July 2009.

[2] A. Shacham, K. Bergman and L.P. Carloni, "On the Design of a Photonic Network-on-Chip", Proceedings of the First International Symposium on Networks-on-Chip (NOCS '07).

[3] N. Sherwood-Droz, H. Wang, L. Chen, B. G. Lee, A. Biberman, K. Bergman, and M. Lipson, "Optical $4 \times 4$ hitless silicon router for optical networks-on-chip (NoC)," Opt. Express, vol. 16, pp. 15915-15922, 2008.

[4] A. Biberman, N. Sherwood-Droz, B. G. Lee, M. Lipson, and K. Bergman, "Thermally active $4 \times 4$ non-blocking switch for networkson-chip," in Proc. Annu. Meeting Lasers and Electro-Optics Society (IEEE), Newport Beach, CA, 2008, pp. 370-371, Paper TuBB3.

[5] B. G. Lee, A. Biberman, K. Bergman, N. Sherwood-Droz, and M. Lipson, "Multi-wavelength message routing in a non-blocking fourport bidirectional switch fabric for silicon photonic networks-on-chip," in Proc. Optical Fiber Communications Conf. (IEEE), San Diego, CA, 2009, pp. 1-3, Paper OMJ4

[6] A. Parini, G. Bellanca, A. Annoni, F. Morichetti, A. Melloni, M.J. Strain, M. Sorel, C. Pareige, M. Gay, L. Bramerie, and M. Thual, "Bit Error Rate Performance Evaluation of a Silicon-On-Insulator Optical-Network-On-Chip Router in a WDM Configuration". in European Conference and Exhibition on Optical Communication (ECOC) (The Institution of Engineering and Technology 2013), P.2.4.

[7] X. Tan, M. Yang, L. Zhang, Y. Jiang, and J. Yang, "On a Scalable, Non-Blocking Optical Router for Photonic Networks-on-Chip Designs", Photonics and Optoelectronics (SOPO), 2011 Symposium on.

[8] M. Gnan, S. Thoms, D. S. Macintyre, R. M. De La Rue, and M. Sorel, Electron. Lett. 44, 115-116, 2008.

[9] $\quad$ ASPIC $^{\circledR}:$ http://www.aspicdesign.com

[10] D. Melati, F. Morichetti, A. Canciamilla, D. Roncelli, F. M. Soares, A. Bakker, and A. Melloni, "Validation of the building block based approach for the design of photonic integrated circuits," J. Lightw. Technol., vol. 30, no. 23, pp. 3610-3616, Dec. 2012.

[11] T.N. Nguyen, M. Gay, K. Lenglé, L. Bramerie, M. Thual, J.C. Simon, S. Malaguti, G. Bellanca, S. Trillo, S. Combrié, G. Lehoucq, and A. De Rossi, " 100-Gb/s Wavelength Division Demultiplexing Using a Photonic Crystal Four-Channel Drop Filter", IEEE Photonics Technology Letters, vol. 25, no. 9, may 1, 2013.

[12] A. Canciamilla, F. Morichetti, S. Grillanda, P. Velha, M. Sorel, V. Singh, A. Agarwal, L.C. Kimerling, and A. Melloni, "Photo-induced trimming of chalcogenide-assisted silicon waveguides," Opt. Express, vol. 20, pp. 15807-15817, 2012.

[13] D. Bachman, Z. Chen, R. Fedosejevs, Y. Tsui, and V. Van, "Permanent fine tuning of silicon microring devices by femtosecond laser surface amorphization and ablation," Opt. Express 21, pp. 11048-11056, 2013. 